\title{
Clinical relevance of Wilms tumor 1 gene mutations in childhood acute myeloid leukemia
}

Iris H. I. M. Hollink, Marry M. van den Heuvel-Eibrink, Martin Zimmermann, Brian V. Balgobind, Susan T. C. J. M. Arentsen-Peters, Marielle Alders, Andre Willasch, Gertjan J. L. Kaspers, Jan Trka, Andre Baruchel, Siebold S. N. de Graaf, Ursula Creutzig, Rob Pieters, Dirk Reinhardt and C. Michel Zwaan

Updated information and services can be found at:

http://bloodjournal.hematologylibrary.org/content/113/23/5951.full.html

Articles on similar topics can be found in the following Blood collections Myeloid Neoplasia (523 articles)

Information about reproducing this article in parts or in its entirety may be found online at: http://bloodjournal.hematologylibrary.org/site/misc/rights.xhtml\#repub_requests

Information about ordering reprints may be found online at:

http://bloodjournal.hematologylibrary.org/site/misc/rights.xhtml\#reprints

Information about subscriptions and ASH membership may be found online at: http://bloodjournal.hematologylibrary.org/site/subscriptions/index.xhtml

Blood (print ISSN 0006-4971, online ISSN 1528-0020), is published weekly by the American Society of Hematology, 2021 L St, NW, Suite 900, Washington DC 20036.

Copyright 2011 by The American Society of Hematology; all rights reserved.

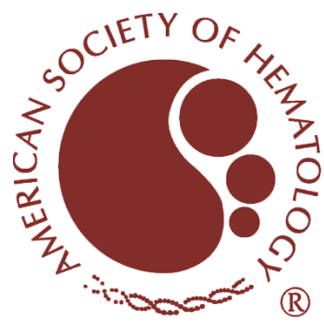




\section{Clinical relevance of Wilms tumor 1 gene mutations in childhood acute myeloid leukemia}

Iris H. I. M. Hollink, ${ }^{1}$ Marry M. van den Heuvel-Eibrink, ${ }^{1}$ Martin Zimmermann, ${ }^{2}$ Brian V. Balgobind, ${ }^{1}$ Susan T. C. J. M. Arentsen-Peters, ${ }^{1}$ Marielle Alders, ${ }^{3}$ Andre Willasch, ${ }^{4}$ Gertjan J. L. Kaspers, ${ }^{5}$ Jan Trka, ${ }^{6}$ Andre Baruchel, ${ }^{7}$ Siebold S. N. de Graaf, ${ }^{8}$ Ursula Creutzig, ${ }^{9}$ Rob Pieters, ${ }^{1}$ Dirk Reinhardt, ${ }^{2}$ and C. Michel Zwaan ${ }^{1}$

${ }^{1}$ Department of Pediatric Oncology/Hematology, Erasmus MC-Sophia Children's Hospital, Rotterdam, The Netherlands; ${ }^{2}$ AML-BFM Study Group, Department of Pediatric Oncology/Hematology, Medical High School, Hannover, Germany; ${ }^{3}$ Clincial Genetics, Academic Medical Center, Amsterdam, The Netherlands; ${ }^{4}$ Department of Hematology and Oncology, University Children's Hospital of Frankfurt, Frankfurt, Germany; ${ }^{5}$ Department of Pediatric Oncology/Hematology, VU University Medical Center, Amsterdam, The Netherlands; ${ }^{6}$ Pediatric Haematology/Oncology, Second Medical School, Charles University Prague, Prague, Czech Republic; ${ }^{7}$ Hematology, Saint-Louis Hospital, Paris, France; ${ }^{8}$ Department of Pediatric Oncology/Hematology, Radboud University Medical Center, Nijmegen, The Netherlands; and ${ }^{9}$ AML-BFM Study Group, University Children's Hospital, Münster, Germany

Wilms tumor 1 (WT1) mutations have recently been identified in approximately $10 \%$ of adult acute myeloid leukemia (AML) with normal cytogenetics (CN-AML) and are associated with poor outcome. Using array-based comparative genome hybridization in pediatric CN-AML samples, we detected a WT1 deletion in one sample. The other WT1 allele was mutated. This prompted us to further investigate the role of $W T 1$ aberrations in childhood AML. Mutations were found in 35 of 298 (12\%) diagnostic pediatric AML samples. In 19 of 35 (54\%) samples, more than one WT1 aberration was found: 15 samples had 2 different mutations, 2 had a homozygous mutation, and 2 had a mutation plus a WT1 deletion. WT1 mutations clustered significantly in the $\mathrm{CN}$-AML subgroup (22\%; $P<.001)$ and were associated with FLT3/ITD (43 vs $17 \% ; P<.001$ ). WT1 mutations conferred an independent poor prognostic significance (WT1 mutated vs wildtype patients: 5 -year probability of overall survival [pOS] $35 \%$ vs $66 \%, P=.002$; probability of event-free survival $22 \%$ vs $46 \%, P<.001$; and cumulative incidence of relapse or regression $70 \%$ vs $44 \%$, $P<.001)$. Patients with both a $W T 1$ mutation and a FLT3/ITD had a dismal prognosis (5-year pOS 21\%). WT1 mutations occur at a significant rate in childhood $A M L$ and are a novel independent poor prognostic marker. (Blood. 2009;113: 5951-5960)

\section{Introduction}

Childhood acute myeloid leukemia (AML) is a heterogeneous disease characterized by different recurrent cytogenetic aberrations that, together with early treatment response, determine the current risk-group classification in childhood AML. ${ }^{1,2}$ Over the past decades, the prognosis of childhood AML patients has improved significantly, now reaching long-term survival rates of approximately $60 \% .^{3}$ Further intensification of chemotherapy is not expected to improve survival any further, as the current regimens are already very intensive, resulting in relatively high frequencies of treatment-related death, as well as long-term side effects. ${ }^{1}$ Instead, new therapeutic strategies are needed. Drugs that target specific aberrations in the leukemic cells are of advantage, not only for improving prognosis, but also as they may cause less side effects. Therefore, the identification of new genetic aberrations that play a role in the pathogenesis of pediatric AML is warranted. Such aberrations may also improve classification and risk-group stratification.

In childhood AML, approximately $20 \%$ to $25 \%$ of cases at diagnosis cannot be characterized by karyotypic aberrations and are referred to as cytogenetically normal AML (CN-AML). In adults, this subgroup is larger (approximately 40\%-50\%), and several molecular aberrations have been identified in this subgroup, such as mutations in NPM1,
$C E B P \alpha$, partial tandem duplications of the $M L L$ gene (MLL-PTD), as well as internal tandem duplications of the FLT3 gene (FLT3/ITD). These molecular aberrations are also found in childhood AML, but in a smaller percentage of childhood CN-AML compared with adult CN-AML. In part, this is due to a 2-fold lower frequency of NPMI mutations in children $(20 \%-25 \%)^{4-6}$ compared with adults $(50 \%-60 \%){ }^{7}$ Thus, in a relatively large cohort of childhood CN-AML, the underlying genetic aberrations are still unknown.

To identify novel abnormalities in childhood CN-AML, we performed array-based comparative genome hybridization (array$\mathrm{CGH}$ ), and found an 11p13 deletion that included the Wilms tumor 1 (WT1) gene in 1 of 43 patients (Figure 1A). The remaining WT1 allele carried a truncating mutation (Figure 1B). This observation focused our interest on WT1 aberrations in childhood AML, as only limited data on this topic are available..$^{8,9}$

The WT1 gene is known to be overexpressed in leukemias and, therefore, is used as a marker for minimal residual disease (MRD) detection, as well as a target for immunotherapy. ${ }^{10}$ The gene encodes for a zinc-finger-motif-containing transcription factor involved in the regulation of growth and differentiation. Although the precise role of the WT1 protein in hematopoieses is currently
Submitted September 7, 2008; accepted January 7, 2009. Prepublished online as Blood First Edition paper, January 26, 2009; DOI 10.1182/blood-2008-09177949.

An Inside Blood analysis of this article appears at the front of this issue.
The online version of this article contains a data supplement.

The publication costs of this article were defrayed in part by page charge payment. Therefore, and solely to indicate this fact, this article is hereby marked "advertisement" in accordance with 18 USC section 1734.

(C) 2009 by The American Society of Hematology 
A

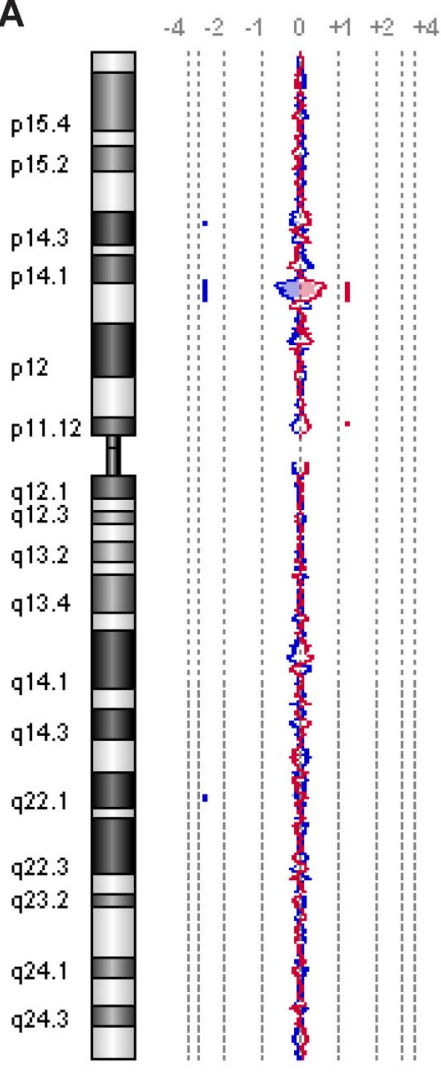

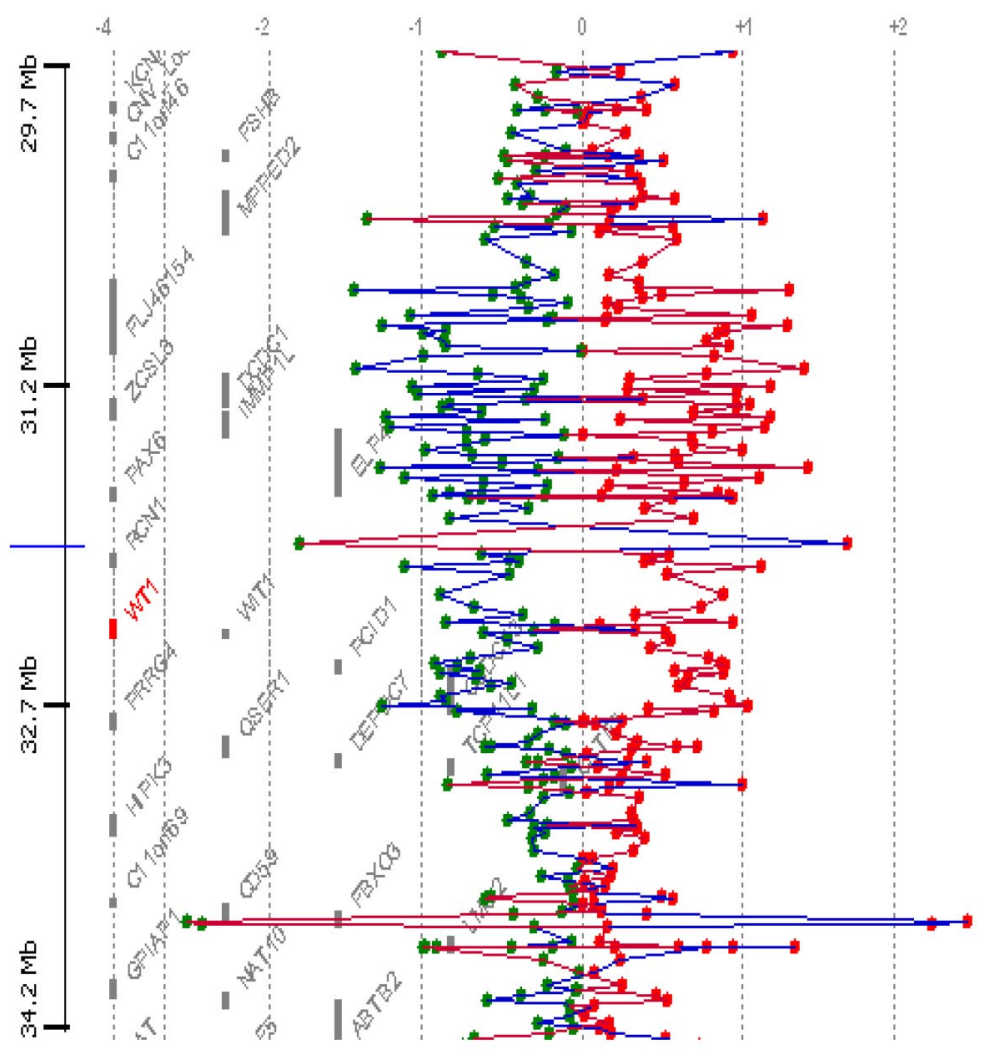

B

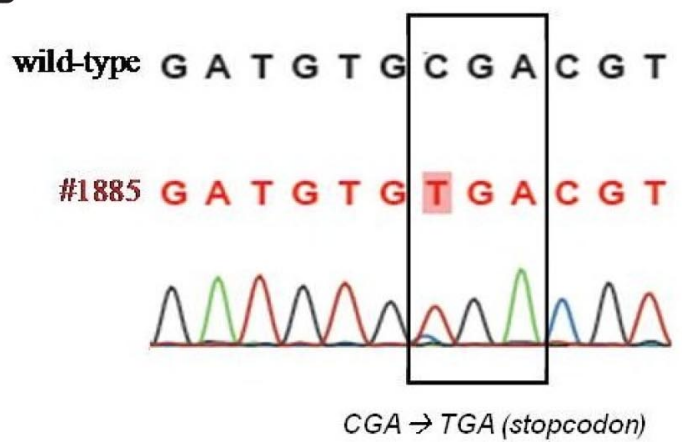

Figure 1. WT1 aberrations in patient no. 1885 detected by array-CGH and sequencing. (A) The left part of the figure shows a chromosome 11 ideogram and corresponding oligonucleotide array-CGH plot of the ratio of patient no. 1885 DNA and control DNA (blue tracing) versus the dye-swap experiment (red tracing) from an array-CGH experiment (105-K oligonucleotide array-CGH platform; Agilent Technologies, Palo Alto, CA). A cryptic 11p13 deletion of approximately $2.5 \mathrm{Mb}$ is found, which area includes the WT1 gene. The right part of the figure zooms in on the deleted area and presents the genes located in this area. (B) Sequence analysis of patient no. 1885 showing a c.901C $\rightarrow$ T non-sense mutation on the remaining WT1 allele.

unknown, it has been hypothesized to act both as an enhancer of cellular quiescence in hematopoietic stem cells and as an inducer of cellular differentiation in more committed precursor cells. ${ }^{10,11}$ WT1 mutations cause translation of an aberrant protein with loss of normal function and might therefore result in stem cell proliferation and blocking of differentiation, thereby contributing to leukemogenesis. Recently, WT1 mutations were found in approximately $10 \%$ of the adult CN-AML subgroup and were reported to be associated with treatment failure and a poor prognosis. ${ }^{12-14}$

To determine further the role of WT1 aberrations in childhood AML, we searched for these aberrations in a large, wellcharacterized cohort of childhood AML patients. WT1 mutations were found in $12 \%$ of diagnostic childhood AML samples and appeared to confer independent prognostic significance.

\section{Methods}

\section{Patient samples}

Viably frozen bone marrow (BM) or peripheral blood (PB) samples taken at diagnosis from 298 children with AML were provided by the Dutch Childhood Oncology Group (DCOG; The Hague, The Netherlands), the AML-Berlin-Frankfurt-Münster Study Group (AML-BFM-SG; Hannover, Germany, and Prague, Czech Republic), and the Saint-Louis Hospital (Paris, France). In addition, 39 paired diagnostic-relapse samples (BM or $\mathrm{PB})$ and 5 paired diagnostic-remission samples (BM) were provided by the DCOG and AML-BFM-SG. Institutional review board approval for these studies had been obtained from Erasmus MC-Sophia Children's Hospital according to local laws and regulations, and informed consent was obtained accordance with the Declaration of Helsinki. Each national study group 
performed central review of the morphologic and cytogenetic classification, as well as clinical follow-up. For a complete list of the individual study participants in the DCOG and AML-BFM studies, see the Appendix (available on the Blood website; see the Supplemental Materials link at the top of the online article).

After thawing of the samples, contaminating nonleukemic cells were eliminated as previously described..$^{15}$ Blast percentages were assessed morphologically on May-Grünwald-Giemsa-stained cytospin slides. Genomic DNA and total cellular RNA were extracted from leukemic cells using TRIzol reagent (Invitrogen, Breda, The Netherlands) as described before. ${ }^{16}$

Samples were routinely screened using standard chromosome banding analysis by the national study groups and were analyzed further in our laboratory for recurrent nonrandom genetic abnormalities, including $\mathrm{t}(15 ; 17), \operatorname{inv}(16), \mathrm{t}(8 ; 21)$, and $M L L$ gene rearrangements, using reverse transcription polymerase chain reaction (RT-PCR) and/or fluorescent in situ hybridization.

\section{Treatment protocols}

WT1 mutation analysis was performed on samples obtained from patients who were treated between 1987 and 2005, according to consecutive AML-BFM-SG/DCOG and to Leucámie Aiquë Myéloïde Enfant (LAME) collaborative childhood AML treatment protocols. The survival analysis was, however, restricted to the patients included in the AML-BFM-SG/ DCOG cohorts, to reduce the variability in treatment regimens, and because this comprised the majority of patients $(n=232)$. Details of the treatment protocols included in the survival analysis and overall outcome data have been previously published, with the exception of study AML-BFM 04, which is ongoing. ${ }^{17-19}$ In these protocols, treatment consisted of 4 to 5 blocks of intensive chemotherapy, using a standard cytarabine and anthracycline backbone. Stem cell transplantation (SCT) in CR1 was used only in selected high-risk patients.

\section{Mutation analysis of $W T 1$}

For mutation analysis of exons 7 through 10 of the WT1 gene, genomic DNA was PCR-amplified using specific primers described in Table S1. The following PCR conditions were used: 2 minutes at $50^{\circ} \mathrm{C}, 10$ minutes at $95^{\circ} \mathrm{C}, 40$ cycles of 15 minutes at $95^{\circ} \mathrm{C}$ and 1 minute at $60^{\circ} \mathrm{C}$, and an extension step of 10 minutes at $72^{\circ} \mathrm{C}$. Purified PCR products were directly sequenced from both strands using the described primers. The sequence data were analyzed using CLC Workbench version 3.5.1 (CLC Bio, Aarhus, Denmark). WT1 mutants without an unambiguously identified sequence or containing more than one WT1 mutation were confirmed by cloning using the TOPO-TA Cloning kit (Invitrogen). Plasmid DNA from multiple isolated clones was purified and directly sequenced from both strands.

To explore the presence of mutations in the other exons of the WT1 gene, exons 1-6 were screened in a cohort of 68 patients, which included 28 patients with WT1 mutations in exons 7-10 and 40 patients with wild-type WT1 for exons 7-10. For mutation analysis of exons 1-6, the purified DNA was subjected to 41 cycles of PCR using a touchdown approach of 30 minutes at $94^{\circ} \mathrm{C}, 30$ minutes at $66^{\circ}-54^{\circ} \mathrm{C}\left(1 \times 66^{\circ} \mathrm{C}\right.$, $2 \times 64^{\circ} \mathrm{C}, 3 \times 62^{\circ} \mathrm{C}, 4 \times 60^{\circ} \mathrm{C}, 5 \times 58^{\circ} \mathrm{C}, 6 \times 56^{\circ} \mathrm{C}$, and $\left.20 \times 54^{\circ} \mathrm{C}\right)$ and 30 minutes at $72^{\circ} \mathrm{C}$ using (M13-tagged) primers also described in Table S1. PCR products were directly sequenced unidirectionally using M13-primers and analyzed using Codoncode aligner (Codoncode, Dedham, MA). Mutations were confirmed by an independent amplification of the fragment and direct sequencing of both strands. This method used for analysis of exons 1 to 6 has originally been developed for mutation detection in nephroblastomas.

\section{Expression analysis of mutated $W T 1$}

To confirm the expression of mutated WT1, cDNA of WT1 exon 7-mutated samples was PCR-amplified using specific primers described in Table S1. PCR and direct sequencing conditions were the same as for WT1 mutation analysis on genomic DNA as described above.

For quantitative expression analysis, quantitative RT-PCR of mutated WT1 samples was performed as described before. ${ }^{20,21}$

\section{Multiplex ligation-dependent probe amplification}

Multiplex ligation-dependent probe amplification (MLPA) was used to detect small deletions in the WT1 gene. MLPA analysis was performed using the SALSA MPLA Kit P118 (MRC Holland, Amsterdam, The Netherlands). SALSA P118 consists of one reaction mixture containing probes for all WTI exons and 20 control probes in other regions of the genome. The exact location of the MLPA probes can be downloaded from the MRC Holland website (http://www.mrc-holland.com). MPLA was carried out according to the manufacturer's protocol. Electrophoresis of MLPA-PCR products was done on an ABI-Prism 310 (Applied Biosystems, Foster City, CA). Data analysis was performed by exporting the peak areas to Microsoft Excel. Sample-related and peak-related differences in PCR and electrophoresis efficiency were corrected by first calculating the peak area relative to the sum of peak areas per sample and subsequently calculating each normalized peak area relative to the mean of that peak in control samples. Peak heights outside the range 0.7 to 1.3 times the control peak height were considered abnormal, with those below 0.7 representing deletions, and those above 1.3 representing duplications. Several control samples were included in each MLPA test.

\section{Mutation analysis of other molecular aberrations}

Detection of other molecular aberrations, including mutations of $C E B P \alpha$, FLT3/ITD and TKD, NPMI, N-RAS and K-RAS, PTPN11 and $c-K I T$, was performed as previously described. ${ }^{6,22-25}$ The detection of $M L L$-PTD was done with PCR as previously described ${ }^{26}$ and confirmed with MLPA (MRC Holland). The reaction mixture for MPLA-analysis contained probes for exons 2 through 13 of the $M L L$ gene and exon 17 as internal control. A probe in the serpinB2 gene was used as external control (manuscript submitted; primers available on request). MLPA was performed according to the manufacturer's protocol. Data were analyzed using GeneMarker version 1.5 (SoftGenetics, State College, PA).

\section{Statistical analysis}

To compare categorical variables we used $\chi^{2}$ analysis and the Fisher exact test for small patient numbers. The nonparametric Mann-Whitney U test was applied for continuous variables.

To assess outcome, the following parameters were used: complete remission $(\mathrm{CR})$ rate, resistant disease ( $\mathrm{RD}$; defined as failure to achieve remission but excluding patients with early death), probability of event-free survival (pEFS; defined as time between diagnosis and first event, including relapse, death by any cause, failure to achieve remission or second malignancy), probability of overall survival (pOS; defined as time between diagnosis and death from any cause), and the cumulative incidence of relapse or nonresponse (CIR; defined as time between diagnosis and relapse; resistant disease was included as an event on day 0). pEFS and pOS were estimated by the Kaplan-Meier method and compared using the log-rank test. The CIR curves were constructed by the method of Kalbfleisch and Prentice and were compared using Gray test. The independence of prognostic factors was examined by multivariate Cox regression analysis.

$P$ values less than .05 were considered statistically significant (2-tailed testing).

\section{Results}

\section{Study population}

We screened 298 diagnostic childhood AML samples for WT1 mutations. The patient characteristics are shown in Table 1. Survival analysis was restricted to the patients who were treated using uniform protocols $(n=232)$; hence patients treated according to other protocols were excluded $(n=39)$. In addition, patients with promyelocytic leukemia-retinoic acid receptor $\alpha$ (PMLRAR $\alpha ; n=15)$, secondary AML $(n=8)$, treatment without curative intent $(n=1)$, and patients lost to follow-up $(n=3)$ were 
Table 1. Clinical and genetic characteristics of the 298 childhood AML patients included in this study, divided by WT1 mutational status

\begin{tabular}{|c|c|c|c|c|}
\hline & All patients & WT1 mutated & WT1 wild-type & $P$ \\
\hline Number (\%) & $298(100 \%)$ & $35(11.7 \%)$ & 263 (88.3\%) & \\
\hline Median age, $y$ & 9.7 & 9.2 & 9.7 & .34 \\
\hline$<3 y, n(\%)$ & 60 & $1(2 \%)$ & $59(98 \%)$ & \\
\hline$\geq 3$ and $<10 y, n(\%)$ & 93 & $17(18 \%)$ & $76(82 \%)$ & .008 \\
\hline$\geq 10 y, n(\%)$ & 145 & $17(12 \%)$ & $128(88 \%)$ & \\
\hline Sex (\% female) & $46.6 \%$ & $48.6 \%$ & $46.4 \%$ & .81 \\
\hline WBC, $\times 10^{9} / \mathrm{L}$, median (range) & $38.0(0.0-534.6)$ & $57.2(2.5-332.0)$ & $34.1(0.0-534.6)$ & .007 \\
\hline FAB, $n(\%)$ & & & & .60 \\
\hline MO & $15(5 \%)$ & $4(12 \%)$ & $11(4 \%)$ & \\
\hline M1 & $37(13 \%)$ & $6(18 \%)$ & $31(12 \%)$ & \\
\hline M2 & $66(23 \%)$ & $7(21 \%)$ & $59(23 \%)$ & \\
\hline M3 & $21(8 \%)$ & $2(6 \%)$ & $19(8 \%)$ & \\
\hline M4 & $72(25 \%)$ & $8(24 \%)$ & $64(25 \%)$ & \\
\hline M5 & $61(21 \%)$ & $7(21 \%)$ & $54(21 \%)$ & \\
\hline M6 & $6(2 \%)$ & $0(0 \%)$ & $6(2 \%)$ & \\
\hline M7 & $9(3 \%)$ & $0(0 \%)$ & $9(4 \%)$ & \\
\hline Other & $2(1 \%)$ & $0(0 \%)$ & $2(1 \%)$ & \\
\hline Unknown & $9(3 \%)$ & $1(3 \%)$ & $8(3 \%)$ & \\
\hline FLT3/ITD $(\mathrm{n}=298), \mathrm{n}(\%)$ & $60(20 \%)$ & $15(43 \%)$ & $45(17 \%)$ & $<.001$ \\
\hline Median allelic ratio (range) & $0.59(0.21-2.33)$ & $0.69(0.32-1.25)$ & $0.58(0.21-2.33)$ & .22 \\
\hline Median ITD length (range) & $54(18-209)$ & $51(18-90)$ & $55(20-209)$ & .44 \\
\hline$N$ - or $K-R A S(\mathrm{n}=282), \mathrm{n}(\%)$ & $63(22 \%)$ & $8(25 \%)$ & $55(22 \%)$ & .70 \\
\hline$c-K I T(\mathrm{n}=283), \mathrm{n}(\%)$ & $18(6 \%)$ & $0(0 \%)$ & $18(7 \%)$ & .24 \\
\hline$C E B P \alpha(n=250), n(\%)$ & $20(8 \%)$ & $5(19 \%)$ & $15(7 \%)$ & .03 \\
\hline$M L L-P T D(\mathrm{n}=251), \mathrm{n}(\%)$ & $6(2 \%)$ & $0(0 \%)$ & $6(3 \%)$ & $>.999$ \\
\hline NPM1 ( $\mathrm{n}=293), \mathrm{n}(\%)$ & $24(8 \%)$ & $0(0 \%)$ & $24(9 \%)$ & .09 \\
\hline
\end{tabular}

WBC indicates white blood cell count at diagnosis; and FAB, French-American-British morphology classification.

excluded. The WT1-mutated and WT1 wild-type AML patients were equally distributed over the different treatment protocols $(P=.43)$, and there was no significant difference between the treatment protocols in terms of pEFS and pOS (respectively, $P=.75$ and $P=.38$ ). The patient characteristics of the cohort used for survival analysis are shown in Table $\mathrm{S} 2$.

\section{WT1 mutation analysis}

We first analyzed the samples for mutations in the hotspot areas exons 7-10 of the WT1 gene. A total of 48 WT1 gene mutations were detected in 34 of the 298 (11.4\%) samples. These mutations predominantly clustered in 2 areas in exon $7(n=41)$, but were also found in exon $8(n=1)$ and exon $9(n=4$; Table 2$)$. In 2 cases, WT1 mutations were detected in intron 6-7, located on the splice-acceptor site; these mutations are predicted to disrupt the splice site of exon 7 and are therefore considered pathogenic. Subsequently, a subset of 68 patients was also analyzed for mutations in exons 1 to 6 , and additional mutations were found in exon $1(\mathrm{n}=2)$, exon $2(\mathrm{n}=1)$, and exon $3(\mathrm{n}=2)$. The mutations in exons 1,2 , and 3 occurred only in the samples harboring an exon 7 mutation as well, except for one sample that carried both an exon 2 and 3 mutation. Therefore, the total number of mutations detected in the WT1 gene was 53 mutations in 35 of 298 (11.7\%) samples. The locations and hotspots of the WT1 mutations are presented schematically in Figure 2.

Most of the WT1 mutations were frame-shift mutations $(\mathrm{n}=41)$ predominantly caused by insertions (range, 1-76 bp) but also by deletions (range, 1-32 bp). Six mutations were non-sense mutations. The frame-shift and non-sense mutations are predicted to result in truncated proteins and, when occurring in exon 7, to the loss of the zinc-finger DNA-binding motif of the WT1 protein. The remaining mutations $(n=6)$ were mis- sense mutations leading to substitutions of single amino acids. One of these occurred in exon 9 (c.1186G $>A$ ), which is a known pathogenic mutation in the Denys-Drash syndrome. ${ }^{27}$ The other 5 missense mutations occurred in exon 7, besides a frame-shift mutation in exon 7.

Apart from the 53 pathogenic mutations, we also detected mutations that we considered to be nonpathogenic. In 3 cases, silent mutations, which do not result in amino acid changes, were found. In 6 patients, an identical single base-pair substitution was detected in intron 6-7, which is located 9 nucleotide positions before the start of exon 7 (c.895-9T $>$ C). However, both the lack of a transcript variant in these patients (data not shown) and the analysis of this substitution with regard to the splice-donor and spliceacceptor sites indicated no influence on splicing. These mutations were therefore regarded as nonpathogenic. In one patient, a missense mutation in exon 1 (c.124C $>$ T), causing the substitution of the amino acid proline by a serine, was found. No germ line sample or material of the parents was available to test the leukemic-specific origin of this mutation. However, neither this mutation nor the affected codon has been described before in malignancies. Therefore, we regarded this mutation as an unclassified variant. Several known single-nucleotide polymorphisms (SNPs) were found. One known $\operatorname{SNP}($ c.903A $>$ G) is situated in the mutational hotspot of exon 7 and was detected in 72 of the 298 diagnostic samples (24\%). The frequency of this SNP was not different between AML samples harboring a WT1 mutation compared with WT1 wild-type samples (respectively, 8/35 [23\%] vs 64/263 [24\%]).

\section{WT1 microdeletions}

To evaluate microdeletions of the WT1 gene, which might act as a mechanism of knockdown of the other WT1 allele, we used the MLPA 
From bloodjournal.hematologylibrary.org at Erasmus MC Medical Library on August 9, 2011. For personal use BLOOD, 4 JUNE 2009 • VOLUME 113, NUMBER 23

Table 2. Characteristics of the WT1 aberrations detected in the diagnostic samples of 35 childhood AML patients

\begin{tabular}{|c|c|c|c|c|c|}
\hline UPN & Mutation(s) $^{\star}$ & Exon & Type of mutation & $\begin{array}{l}\text { Protein } \\
\text { level† }\end{array}$ & $\begin{array}{l}\text { More than one } \\
\text { allele affected? }\end{array}$ \\
\hline \multirow[t]{3}{*}{1} & c.905delGinsCC & 7 & frame-shift & & yes \\
\hline & c.902_938dup37 & 7 & frame-shift & & \\
\hline & c. $943 \mathrm{~T}>\mathrm{C}$ & 7 & missense & p.Ser316Pro & \\
\hline \multirow[t]{2}{*}{2} & c. $.901 \mathrm{C}>\mathrm{T}$ & 7 & nonsense & p.Arg301X & yes \\
\hline & del11p13, including WT1‡ & & & & \\
\hline \multirow[t]{2}{*}{3} & c.934_935insA & 7 & frame-shift & & yes \\
\hline & c. $979 \mathrm{~T}>\mathrm{C}$ & 7 & missense & p.Tyr327His & \\
\hline 4 & c. [920_932del13;934C>G] & 7 & frame-shift & & no \\
\hline \multirow[t]{2}{*}{5} & c. $524 \mathrm{C}>\mathrm{A}$ & 2 & nonsense & p.Ser175X & unknown \\
\hline & c.584_585insCCGG & 3 & frame-shift & & \\
\hline \multirow[t]{2}{*}{6} & c. $938 \mathrm{C}>\mathrm{A}$ & 7 & nonsense & p.Ser313X & yes \\
\hline & c.935_939dupCGGTC & 7 & frame-shift & & \\
\hline 7 & c.938_939dupTC & 7 & frame-shift & & no \\
\hline 8 & c.905delGinsCC & 7 & frame-shift & & no \\
\hline 9 & c.934_1009dup76 & 7 & frame-shift & & no \\
\hline 10 & c.[901C>T;904_905insGA] + [901C>T;904_905insGA] & 7 & nonsense plus frame-shift & p.Arg301X & yes \\
\hline \multirow[t]{2}{*}{11} & c.933delA & 7 & frame-shift & & yes \\
\hline & c.1188_1189ins17 & 9 & frame-shift & & \\
\hline 12 & c. $1186 \mathrm{G}>\mathrm{A}$ & 9 & missense & p.Asp396Asn\| & no \\
\hline 13 & c.937_940dupTCGG & 7 & frame-shift & & no \\
\hline 14 & c.901delCinsGCG & 7 & frame-shift & & no \\
\hline 15 & c.937_940dupTCGG & 7 & frame-shift & & no \\
\hline \multirow[t]{2}{*}{16} & c.905delGinsCC & 7 & frame-shift & & yes \\
\hline & c.926_935del10ins12 & 7 & frame-shift & & \\
\hline \multirow[t]{2}{*}{17} & c.905_906insTT & 7 & frame-shift & & yes \\
\hline & c.895-1_901GGATGTGC>CAACGGG & 7 & frame-shift plus affects splice site & & \\
\hline 18 & c.[937_938insG] + [937_938insG] & 7 & frame-shift & & yes \\
\hline \multirow[t]{2}{*}{19} & c.905_906ins 17 & 7 & frame-shift & & yes \\
\hline & c.924_925insGG & 7 & frame-shift & & \\
\hline 20 & c.934_935insG & 7 & frame-shift & & no \\
\hline \multirow[t]{2}{*}{21} & c.924_925insGGTT & 7 & frame-shift & & yes \\
\hline & c.938_939insG & 7 & frame-shift & & \\
\hline \multirow[t]{2}{*}{22} & c.[933delA;937_939delTCG;1012T>C] & 7 & frame-shift & & yes \\
\hline & c.937_940dupTCGG & 7 & frame-shift & & \\
\hline \multirow[t]{2}{*}{23} & c.593delC & 3 & frame-shift & & unknown \\
\hline & c.901delCinsGG & 7 & frame-shift & & \\
\hline \multirow[t]{2}{*}{24} & c.398ins T & 1 & frame-shift & & unknown \\
\hline & c.907_908insAT & 7 & frame-shift & & \\
\hline 25 & c.[901_902insG;935G >A] & 7 & frame-shift & & no \\
\hline 26 & c.1173_1174insA & 9 & frame-shift & & no \\
\hline \multirow[t]{2}{*}{27} & c. $442 \_442+2 G G T>T T G$ & 1 & affects splice site & & unknown \\
\hline & c.901_902insG & 7 & frame-shift & & \\
\hline \multirow[t]{2}{*}{28} & c.1072_1073insC & 8 & frame-shift & & yes \\
\hline & del 11p13, including WT1§ & & & & \\
\hline 29 & c.937_940dupTCGG & 7 & frame-shift & & no \\
\hline 30 & c.934delCinsGG & 7 & frame-shift & & no \\
\hline 31 & c.933_937dupACGGT & 7 & frame-shift & & no \\
\hline 32 & c. $1168 \mathrm{C}>\mathrm{T}$ & 9 & nonsense & p.Arg390X & no \\
\hline \multirow[t]{3}{*}{33} & c.895-55_895-2del54insCA & intron 6-7 & affects splice site & & yes \\
\hline & c. $938 \mathrm{C}>\mathrm{A}$ & 7 & nonsense & p.Ser313X & \\
\hline & c. $1006 A>G$ & 7 & missense & p.K336G & \\
\hline 34 & c.898_929del32 & 7 & frame-shift & & no \\
\hline 35 & c.900_901insG & 7 & frame-shift & & no \\
\hline
\end{tabular}

UPN indicates unique patient number; nd, not done.

*Mutations are described according to the coding DNA sequence (RefSeq NM_0.46642).

†All frame-shift mutations are predicted to produce truncated proteins.

‡Detected by array-CGH and comfirmed by MLPA.

§Detected by MLPA.

|Mutation is known to be pathogenic in Denys-Drash syndrome.

technique to screen 24 samples (19 WT1-mutated and 5 WT1 wild-type samples) for deletions of exons 1 to 10 . The WT1 deletion already detected by array-CGH (patient 2 in Table 2) was again confirmed by MLPA. One other patient, who appeared to carry a homozygous mutation in exon 8 as detected by sequencing (patient 28 in Table 2), actually harbored a deletion of one WT1 allele. No other sample carried WT1 microdeletions. One patient (patient 9 in Table 2) showed a duplication of exon 7 by MLPA. However, this observation could be explained by an exon 7 mutation in this patient, which consisted of a duplication of $76 \mathrm{bp}$, in which the MLPA probe was exactly situated. 


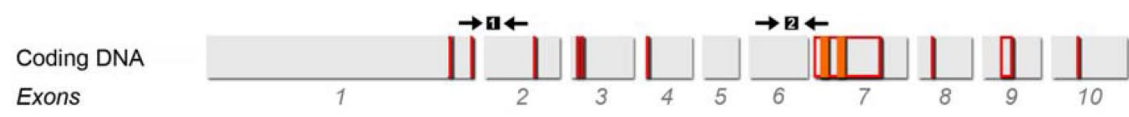

Figure 2. Location of the WT1 gene mutations and their overlap with MRD detection areas. The location of the WT1 gene mutations detected in initial diagnosis and relapse childhood AML samples is schematically presented according to the exon structure of the WT1 gene. Two areas of mutational hotspots were identified in exon 7. Two pairs of primer-probe combinations currently used for WT1-based MRD detection are also depicted in the figure and show overlap with the locations of the Protein domains mutations.
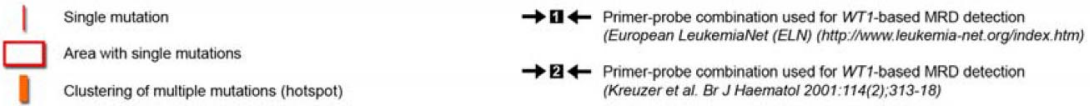

Frequent biallelic involvement of WT1 aberrations

In 19 of 35 (54\%) of the WT1-mutated samples, we detected more than one WT1 aberration. This included either a different WT1 mutation $(\mathrm{n}=15)$, a homozygous WT1 mutation $(\mathrm{n}=2)$, or a deletion of the other $W T 1$ allele $(\mathrm{n}=2$; Table 2$)$. The $W T 1$ deletions were detected by array-CGH and MLPA as described above. Of the 2 patients with homozygous mutations, one was screened with MLPA and did not harbor a deletion, but showed homozygosity of all the SNPs in the $11 \mathrm{p} 13$ area. This suggests that in this patient a loss of heterozygosity has occurred due to uniparental disomy (UPD) of 11p13. Of the 15 samples with more than one different WT1 mutation, 12 samples in fact harbored 2 mutations, and 3 samples each had 3 different mutations. In 11 of the 15 cases, we were able to perform cloning of the PCR products followed by direct sequencing to investigate monoallelic or biallelic involvement of the different mutations. This showed more than one affected allele in 10 of 11 cases. The patient (patient 25 in Table 2), who did not show biallelic involvement of the 2 different mutations, had, in addition to a common frame-shift mutation, a missense mutation in exon 7 on the same allele.

In conclusion, we could show that at least 14 of $35(40 \%)$ of the WT1-mutated samples had more than one WT1 allele affected, that is, 11 cases where both alleles were mutated but with different mutations; 2 cases with a homozygous mutation; and 2 cases with a mutation and a deletion of the other WT1-allele.

\section{Expression of the mutated $W T 1$ gene}

WT1 transcripts of 16 WT1-mutated samples showed the presence of the identical mutations as identified on genomic DNA, indicating that the mutated WT1 gene is expressed at transcriptional level.

Quantitative analysis of WT1 expression was performed in 9 WT1-mutated and 9 WT1 wild-type samples. The median expression (normalized to ABL copies $\times 10000$ ) was $4.1 \times 10^{3}$ (range, $9.1 \times 10^{2}-3.6 \times 10^{4}$ ) in the WT1-mutated samples, which was comparable with the expression measured in the wild-type samples $\left(2.9 \times 10^{3}\right.$; range, $\left.4.8 \times 10^{2}-7.7 \times 10^{3}\right)$. Three of the 9 WT1-mutated samples harbored mutations located exactly at the binding site of the reversed primer used for the quantitative RT-PCR; however, the expression of these samples was still detectable, suggesting that the PCR amplification was relatively unaffected by the mutations in these samples.

\section{Paired diagnostic-remission and diagnostic-relapse samples}

The WT1 mutations were not detectable in the remission material of 5 patients with WT1-mutated AML, hence suggesting somatic origin of the WT1 mutations.

To investigate the clonal stability of WT1 mutations, we screened 39 diagnostic-relapse pairs. This included 11 mutated and
28 wild-type samples at diagnosis. All 11 WT1-mutated diagnostic samples showed the same mutation at relapse. In 2 cases, an additional WT1 mutation was gained, which was situated on the other WT1 allele as determined by cloning. No loss of WT1 mutations was observed. Four of the 28 (14\%) samples gained a WT1 mutation at relapse. Two of these patients in fact gained 2 mutations: in one patient, this concerned mutations in exon 7 and exon 10; in the other patient, in exon 4 and exon 7.

\section{Relationship between WT1 mutations and clinical and genetic characteristics}

The frequency of WT1 mutations was significantly related to age; below the age of 3 years, only 1 of $60(2 \%)$ cases was WT1mutated; in the age group greater than or equal to 3 years and less than 10 years, we found the highest frequency $(17 / 76=18 \%)$; and in children at the age of 10 years or above, the frequency decreased $(17 / 145=12 \% ; P=.008)$. The white blood cell count (WBC) at diagnosis was significantly higher in WT1-mutated AML patients (median $57.2 \times 10^{9} / \mathrm{L}$ ) than in WT1 wild-type AML patients (median $\left.34.1 \times 10^{9} / \mathrm{L} ; P=.007\right)$. No significant differences between the 2 groups were found for sex $(P=.81)$ or for FrenchAmerican-British (FAB)-type distribution $(P=.60)$.

WT1 mutations were not randomly distributed over the different cytogenetic subgroups (Figure 3). Twenty-one of $94(22.3 \%)$ CN-AML cases harbored WT1 mutations compared with 13 of $187(7.0 \%)$ cases with cytogenetic aberrations $(P<.001)$. No mutations were found in the favorable karyotype subgroups $t(8 ; 21)$ and inv(16), and in the $\mathrm{t}(15 ; 17)$ and in the $M L L$-rearranged AML cases, only an occasional WT1 mutation was found. However,

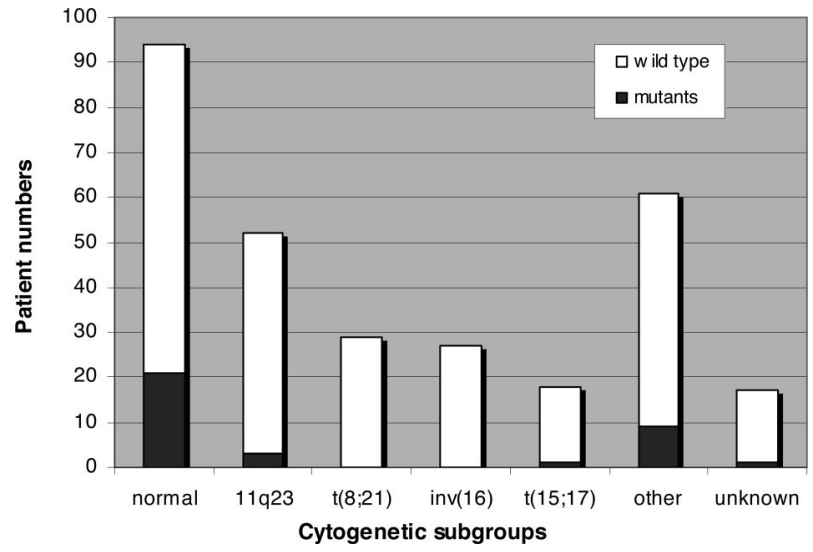

Figure 3. Clustering of the WT1 gene mutations in the subgroup of childhood CN-AML. WT1 mutations mainly clustered in the CN-AML subgroup. No mutations were found in the CBF-AML subgroup [ie, $t(8 ; 21)$ and inv(16)], and very few were detected in the $t(15 ; 17)$ and in the $M L L$ gene-rearranged childhood AML cases. Mutations were also found in the subgroup with other karyotypes, but at a lower frequency $(14.8 \%)$ than in the CN-AML subgroup (22.3\%). 
A

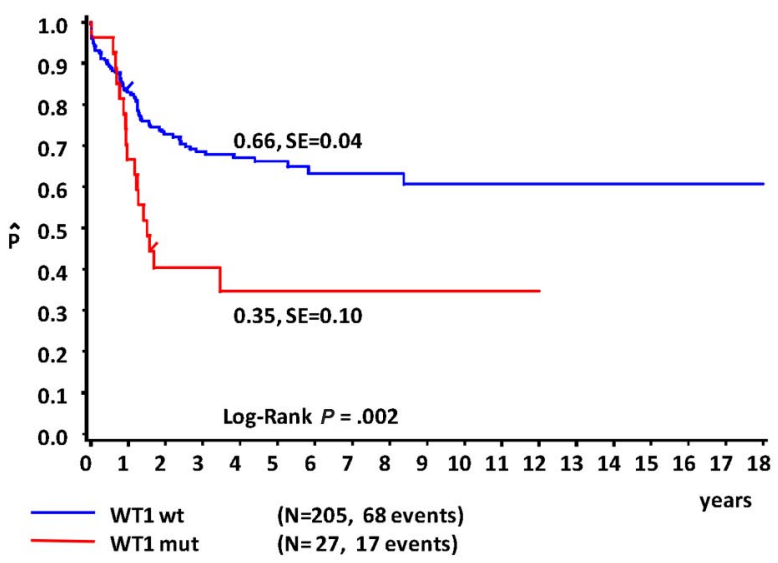

B

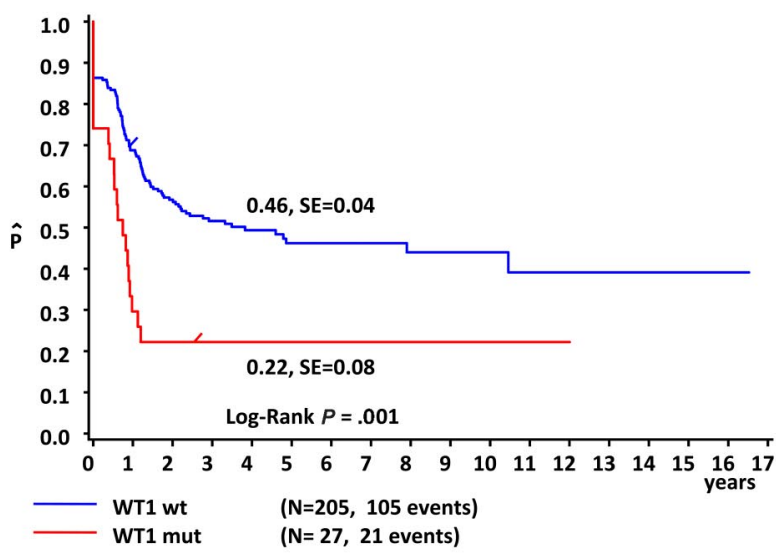

C

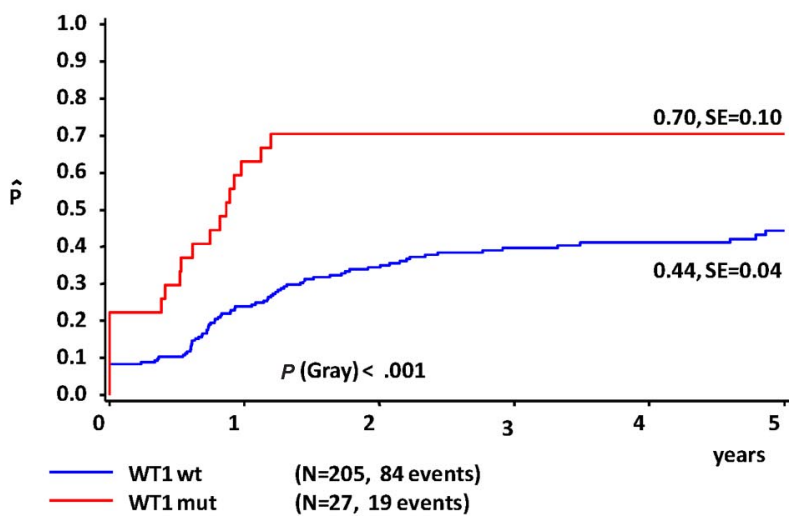

Figure 4. Survival curves of childhood AML patients with and without WT1 mutations. Kaplan-Meier estimates for 5-year pOS (A) and pEFS (B), showing log-rank $P$ values. The 5-year CIR is depicted in $(C)$ and was analyzed according to the Kalbfleisch and Prentice method using the Gray test for statistical significance. WT1-mutated AML patients have a significantly worse outcome in terms of OS, EFS, and CIR compared with patients with WT1 wild-type AML.

within the subgroup with "other cytogenetic aberrations" (defined as all other cytogenetic aberrations than the mentioned subgroups), 9 of $61(14.8 \%)$ samples were WT1-mutated. Two of these 9 patients carried a $\mathrm{t}(6 ; 9)(\mathrm{p} 23 ; \mathrm{q} 34)$ and another 2 patients carried a $9 \mathrm{q}$-deletion. The other patients in this subgroup did not have cytogenetic aberrations in common.

WT1-mutated patients were more likely to harbor a FLT3/ITD (43\% vs $17 \%$ of the $W T 1$ wild-type samples; $P<.001$ ) and a
$C E B P \alpha$ mutation (19\% vs $7 \%$, respectively; $P=.03$; Table 2 ). WT1 and NPM1 mutations were mutually exclusive. WT1 mutations and $c$-KIT mutations and $M L L$-PTD were also mutually exclusive, however, this was based on small numbers only. Within the CN-AML subgroup, we also identified FLT3/ITD to be significantly associated with WT1 mutations (58\% vs 30\% for WT1 mutated and WT1 wild-type patients, respectively; $P=.03$ ). However, no difference in $C E B P \alpha$ mutations (17\% of the WT1-mutated vs $15 \%$ of the $W T 1$ wild-type samples; $P=1.00$ ) was found in the CN-AML subgroup. WT1 mutations and NPM1 mutations were also mutually exclusive in the CN-AML subgroup $(P=.01)$.

The study population included 8 patients with a secondary AML. Two of these patients (1 patient with AML after a preceding MDS and 1 therapy-related AML) harbored a WT1 mutation.

\section{Prognostic impact of WT1 mutations in childhood AML}

The median follow-up time for survivors was 4.4 years ( 4.4 years for WT1 mutated vs 4.4 years for WT1 wild-type AML patients; $P=.89$ ).

The CR rate did not differ significantly between patients with WT1-mutated and WT1 wild-type AML (74.1\% vs $86.3 \%$, respectively; $P=.09)$. However, RD was significantly more frequent in the former group $(23.1 \%$ vs $8.8 \%$, respectively; $P=.03)$. Patients with WT1-mutated AML had a significantly worse pOS, pEFS, and CIR than WT1 wild-type AML patients (5 years pOS $35 \pm 10 \%$ vs $66 \% \pm 4 \% ; P=.002 ;$ pEFS $22 \pm 8 \%$ vs $46 \% \pm 4 \% ; P<.001$; and CIR $70 \% \pm 10 \%$ vs $44 \% \pm 4 \% ; P_{\text {Gray }}<.001$, respectively; Figure 4). The median time to relapse from CR1 was significantly shorter for the WT1-mutated cases (8.5 months) than for the WT1 wild-type cases (11.4 months; $P=.02$ ).

Table 3. Five-year OS according to combined WT1 and FLT3/ITD mutational status

\begin{tabular}{|c|c|c|c|}
\hline \multirow{2}{*}{$\begin{array}{c}\text { FLT3/ITD } \\
\text { status }\end{array}$} & \multicolumn{2}{|c|}{ WT1 mutational status } & \multirow[b]{2}{*}{$\boldsymbol{P}$} \\
\hline & Wild-type & Mutant & \\
\hline Negative & $67 \% \pm 4 \%(n=176)$ & $49 \% \pm 14 \%(n=14)$ & .20 \\
\hline Positive & $63 \% \pm 9 \%(n=29)$ & $21 \% \pm 12 \%(n=13)$ & $<.01$ \\
\hline$P$ & .61 & .09 & \\
\hline
\end{tabular}

Percentages are \pm SE.

Table 4. Five-year EFS according to combined WT1 and FLT3/ITD mutational status

\begin{tabular}{lccr}
\hline \multirow{2}{*}{$\begin{array}{c}\text { FLT3/ITD } \\
\text { status }\end{array}$} & Wild-type & Mutant & \\
\cline { 2 - 3 } & \multicolumn{2}{c}{ WT mutational status } & $\boldsymbol{P}$ \\
\hline Negative & $48 \% \pm 4 \%(\mathrm{n}=176)$ & $29 \% \pm 12 \%(\mathrm{n}=14)$ & .07 \\
Positive & $34 \% \pm 10 \%(\mathrm{n}=29)$ & $15 \% \pm 10 \%(\mathrm{n}=13)$ & $<.01$ \\
$P$ & .32 & .16 & \\
\hline
\end{tabular}

Percentages are \pm SE.

Table 5. Five-year CIR according to combined WT1 and FLT3/ITD mutational status

\begin{tabular}{lccc}
\hline \multirow{2}{*}{$\begin{array}{l}\text { FLT3/ITD } \\
\text { status }\end{array}$} & \multicolumn{2}{c}{ WT1 mutational status } & \\
\cline { 2 - 3 } & Wild-type & Mutant & $\boldsymbol{P}$ \\
\hline Negative & $42 \% \pm 4 \%(\mathrm{n}=176)$ & $64 \% \pm 15 \%(\mathrm{n}=14)$ & .04 \\
Positive & $55 \% \pm 13 \%(\mathrm{n}=29)$ & $77 \% \pm 14 \%(\mathrm{n}=13)$ & .02 \\
$P$ & .27 & .18 & \\
\hline
\end{tabular}

Percentages are \pm SE. 
Table 6. Results of multivariate analysis for OS, EFS, and RFS

\begin{tabular}{llc}
\hline \multicolumn{1}{c}{ Outcome/variable } & HR $(95 \%$ Cl) & $\boldsymbol{P}$ \\
\hline OS & & \\
Other karyotype & $2.83(1.68-4.77)$ & $<.001$ \\
FLT3/ITD & $1.89(1.07-3.34)$ & .03 \\
Favorable karyotype & $0.39(0.15-0.96)$ & .04 \\
$\quad$ WT1 & $1.79(1.02-3.14)$ & .04 \\
EFS & & \\
Other karyotype* & $2.33(1.49-3.64)$ & $<.001$ \\
WT1 & $2.05(1.24-3.38)$ & .005 \\
FLT3/ITD & $1.82(1.14-2.92)$ & .01 \\
Favorable karyotype & $0.74(0.41-1.33)$ & .31 \\
FS & & \\
Other karyotype & & .001 \\
WT1 & $2.44(1.48-4.02)$ & .009 \\
FLT3/ITD & $2.44(1.42-4.17)$ & .58 \\
Favorable karyotype & $1.99(1.19-3.34)$ & \\
\hline
\end{tabular}

WT1 mutations were tested in a Cox regression model with other well-known risk factors in childhood $A M L$ including favorable cytogenetics $t(8 ; 21)$, inv16, other abnormal karyotypes, and FLT3/TD. When including age $>10$ years, WBC $\geq$ $50 \times 10^{9} / \mathrm{L}$, and SCT as time-dependent covariable, the estimates for WT1 mutations were similar, and the $P$ values for these 3 parameters were all $>.10$.

*Other karyotypes indicates all other cytogenetic aberrations than the well-known childhood AML subgroups, that is, normal karyotype and the favorable karyotypes inv(16) and $t(8 ; 21)$

Although numbers were small, there were no differences in CR rate, pOS, pEFS, and CIR between WT1-mutated patients with one $(\mathrm{n}=13)$ or more than one $(\mathrm{n}=14)$ affected allele (data not shown).

As WT1 mutations were frequently associated with FLT3/ITD, we analyzed the impact on outcome according to the combined mutational status (Tables 3-5). Although numbers are small, we found that, both in the FLT3/ITD negative and in the FLT3/ITD positive subgroup, WT1 mutated cases had a worse pEFS and CIR than the WT1 wild-type cases. However, this only translated in a significant survival disadvantage for the WT1 mutated AML patients in the FLT3/ITD positive subgroup; in the FLT3/ITD positive subgroup, the 5 -year pOS was $21 \%( \pm 12 \%)$ for patients with WT1-mutated AML and 63\% $( \pm 9 \% ; P<.01)$ for $W T 1$ wild-type cases. In the FLT3/ITD negative subgroup, these figures were $49 \%( \pm 14 \%)$ versus $67 \%( \pm 4 \% ; P=.20)$. Conversely, we found no significant impact on outcome of FLT3/ITD in the subgroups of WT1 mutated or wild-type AML patients.

\section{Prognostic analysis restricted to the CN-AML subgroup}

In the $\mathrm{CN}$-AML subgroup, the $\mathrm{CR}$ rate was worse in the 19 patients with WT1-mutated AML (74\%) than in the 66 WT1 wild-type AML patients $(91 \% ; P=.05)$. There also was a trend for RD to occur more frequently in patients with WT1 mutated AML (22\% vs 6\%; $P=.07)$. WT1 mutated CN-AML patients had lower pOS and pEFS and higher CIR than WT1 wild-type CN-AML patients (5-year pOS $44 \% \pm 12 \%$ vs $69 \% \pm 6 \%, P=.03 ;$ pEFS $32 \% \pm 11 \%$ vs $49 \pm 6 \%, P=.02$; and CIR $41 \% \pm 7 \%$ vs $58 \% \pm 13 \%, P_{\text {Gray }}=.06$, respectively).

\section{Multivariate analysis}

WT1 mutations were included in a Cox regression model, including age (using 10 years of age as cutoff value), WBC at diagnosis (using $50 \times 10^{9} / \mathrm{L}$ ), cytogenetic subgroups [favorable karyotype, ie, inv(16) and $\mathrm{t}(8 ; 21)$, normal karyotype, and other karyotype], FLT3/ITD, and SCT as time-dependent covariable. We identified WT1 mutations as an independent adverse risk factor for pOS [hazard ratio (HR) 1.79; 95\% confidence interval (CI) 1.02-3.14; $P=.041$ ], pEFS (HR 2.05; 95\% CI 1.24-3.38; $P=.005$ ), as well as for CIR (HR 2.44; 95\% CI 1.42-4.17;
$P=.001$; Table 6). Inclusion of $N P M 1$ and $C E B P \alpha$ mutations in the model did not change the HRs for WT1 mutations. When we entered FLT3/ITD with high allelic ratios (cutoff value $\geq 0.4$ or the median $\geq 0.66$ ) or FLT3/ITD with large ITD length ( $\geq$ median 55 bp) in the model, this did not change the results.

\section{Discussion}

Our study shows that WT1 mutations occur in approximately $12 \%$ of samples from diagnostic childhood AML patients, which is comparable to the frequency found in a smaller study on childhood AML reporting WT1 mutations in 4 of 41 patient samples $(10 \%) .{ }^{8}$ Recently, several large adult AML series were reported, showing frequencies of WT1 mutations ranging from $10 \%$ to $13 \% \cdot{ }^{12-14,28}$ However, these series were restricted to CN-AML. We found a higher percentage in this particular AML subgroup (ie, 22\%). It therefore seems that there is a higher frequency of WT1 gene mutations in childhood AML compared with adult AML. Further support for this difference comes from the typical age pattern that was observed, with a peak frequency in children between the ages of 3 and 10 years. Mutations were sporadically found in children younger than 3 years old, and after the age of 10 years, there was a decreasing frequency. Of interest, 2 other molecular aberrations that also occur frequently within the CN-AML subgroup (ie, NPM1 mutations and FLT3/ITD) both show a different age pattern, as their frequency increases with increasing age., ${ }^{4,6}$ Although not all patients were screened for WT1 mutations in exons 1 to 6 , we do not expect this to significantly increase the mutation frequency, as these mutations were only sporadically found in absence of a $W T 1$ exon 7 mutation.

Our study identifies the presence of WT1 mutations as a novel poor prognostic risk factor for childhood AML. The 5-year overall survival for WT1-mutated AML patients was only $37 \%$ compared with $67 \%$ for the $W T 1$ wild-type AML patients. This was due to a significant increase in the number of events in the WT1-mutated subgroup, including both primary resistant disease, which was found in 23\% of WT1-mutated AML patients, as well as an increased frequency of relapse. This was observed both in the overall group as well as in the CN-AML subset; although some of the differences did not reach statistical significance in the CN-AML subgroup. Multivariate analysis showed that WT1 mutations had strong prognostic significance, independent from other wellknown risk factors in childhood AML, with a 2.4-fold increased risk to relapse despite current intensive chemotherapy. When we combined 2 risk factors (ie, WT1 mutations and FLT3/ITD), a very poor risk group was detected with a 5-year overall survival of only $21 \%$. This indicates an additive effect of carrying both mutations with respect to outcome. Our data need to be prospectively validated, given the retrospective nature of this cell-bank study. However, comparable results regarding outcome have been reported in most, but not all, studies in adults with CN-AML. ${ }^{13,14,28}$

In almost half of the patients WT1 mutations are associated with a FLT3/ITD, which is a well-known poor prognostic factor in childhood AML, especially in case of a high mutant/wild-type allelic ratio (AR). ${ }^{29}$ Moreover, a recent study also showed that the ITD length has prognostic impact in childhood AML. ${ }^{30}$ We did not find differences in the ARs and ITD lengths of the associated FLT3/ITDs between WT1-mutated and wild-type AML patients. WT1 and NPM1 mutations were mutually exclusive and, respectively, predict for poor and excellent outcome in the CN-AML subgroup. ${ }^{4}$ However, when including NPM1 mutations in the multivariate analysis, $W T 1$ mutations still had independent prognostic significance. In the overall cohort, WT1 mutations were also associated with $C E B P \alpha$ mutations; this association disappeared in the $\mathrm{CN}-\mathrm{AML}$ subgroup. As $C E B P \alpha$ mutations are also known to cluster in 
CN-AML, the observed association in the overall group may be biased. The identification of WTI mutations adds to the recognition of $\mathrm{CN}$ $\mathrm{AML}$ as a heterogeneous subgroup with various molecular abnormalities in childhood AML, which may be important for future classification and risk-group stratification in childhood CN-AML.

Mutations in the WT1 gene are localized in 2 hotspot areas in exon 7. However, we also detected mutations in other exons. The mutational hotspots are in agreement with the location of mutations found in previous AML studies, but mutations in exons 4 and 10 have not been reported before. ${ }^{8,9,13,14,28}$ Of interest, the affected mutated areas overlap with different primer-probe combinations used for WT1-based MRD detection, as reported in the literature..$^{21,31,32}$ One of these combinations was used in our study for the expression analysis of the WT1 mutants. ${ }^{21}$ All WT1-mutated samples showed presence of mRNA expression. Surprisingly, expression was also picked up in 3 samples with mutations exactly located at the binding site of the reversed primer, indicating no large effect of these mutations on the PCR amplification, and therewith WT1-based MRD detection. However, a previous case report did show a negative effect on the expression level of WT1 in a sample in which a mutation was gained at the primer binding site. Another potential problem for using WT1 as an MRD marker may be the gain of mutations at relapse, which was observed in $14 \%$ of our relapse samples. Future studies on serial samples taken in the course of the disease need to assess the impact of mutations on MRD detection based on WT1 expression levels.

At least $43 \%$ of the patients with WT1-mutated AML had more than one affected $W T 1$ allele in their leukemic cells. This frequency may be higher, as not all WT1-mutated samples could be screened for additional mutations in exons 1 to 6 . Furthermore, to search for additional second hits in the WT1-mutated AML patients, we also screened for small WT1 deletions using MPLA, but only one extra patient was revealed with a deletion besides a mutation. So far, we were able to detect a second affected allele in approximately half of the WT1-mutated AML patients. Sequence analysis of the cDNA of mutated samples showed expression of the mutated as well as the wild-type transcript in samples with one affected allele. This either suggests a dominant or dominant-negative mechanism of the mutated WT1 protein in the subset of patients with only one affected allele, or other mechanisms of silencing of the wild-type allele such as silencing on RNA level. Epigenetic silencing is unlikely as the wild-type allele is expressed. Of interest, there was no apparent difference in outcome between patients with one affected allele versus those with more than one affected allele. Until now, the precise function of WT1 in hematopoiesis is still unclear, as well as whether
WT1 acts as an oncogene or tumor suppressor gene in AML, which issue was recently reviewed by Yang et $\mathrm{al}^{33}$ Further research is needed to elucidate the underlying functional mechanisms of the mutated WT1 protein and the pathways involved leading to therapy resistance in these patients.

In conclusion, our study showed the presence of WT1 mutations in $12 \%$ of diagnostic childhood AML samples and clustering of these mutations in the CN-AML subgroup (22\%). WT1 mutations are a novel independent poor prognostic factor in childhood AML. In particular, patients with AML carrying both a WT1 mutation and a FLT3/ITD have a very poor outcome of only $21 \%$ 5-year survival in this series. Further exploration of the biology of WT1-mutated AML is necessary to better understand the mechanisms of therapy resistance. This may reveal new potential therapeutic targets for these patients who suffer from a very poor outcome despite current intensive chemotherapy.

\section{Acknowledgments}

I.H.I.M.H. was funded by the Pediatric Oncology Foundation Rotterdam (KOCR), B.V.B. was funded by The Netherlands Organization for Scientific Research (NWO), and D.R. was funded by the Deutsche Krebshilfe.

\section{Authorship}

Contribution: I.H.I.M.H., M.M.v.d.H.-E., and C.M.Z. designed the study; G.J.L.K., J.T., A.B., S.S.N. de G., U.C., and D.R. contributed patient samples and clinical data; I.H.I.M.H., B.V.B., S.T.C.J.M.A.-P., M.A., and A.W. performed the laboratory research; I.H.I.M.H., M.M.v.d.H.-E., R.P., and C.M.Z. analyzed and interpreted the data; M.Z. performed statistical analysis; M.M.v.d.H.-E., A.W., G.J.L.K., J.T., and R.P. reviewed the manuscript; and I.H.I.M.H. and C.M.Z. wrote the manuscript.

Conflict-of-interest disclosure: The authors declare no competing financial interests.

Correspondence: C. Michel Zwaan, Erasmus MC-Sophia Children's Hospital, Department of Pediatric Oncology and Hematology, Dr Molewaterplein 603015 GJ, Rotterdam, The Netherlands; e-mail: c.m.zwaan@erasmusmc.nl.

\section{References}

1. Kaspers GJ, Zwaan CM. Pediatric acute myeloid leukemia: towards high-quality cure of all patients. Haematologica. 2007;92:1519-1532.

2. Rubnitz JE. Childhood acute myeloid leukemia. Curr Treat Options Oncol. 2008;9:95-105.

3. Kaspers GJ, Creutzig U. Pediatric acute myeloid leukemia: international progress and future directions. Leukemia. 2005; 19:2025-2029.

4. Brown P, Mclntyre E, Rau R, et al. The incidence and clinical significance of nucleophosmin mutations in childhood AML. Blood. 2007;110:979-985

5. Cazzaniga G, Dell'Oro MG, Mecucci C, et al. Nucleophosmin mutations in childhood acute myelogenous leukemia with normal karyotype. Blood. 2005;106:1419-1422.

6. Hollink IH, Zwaan CM, Zimmermann M, et al. Favorable prognostic impact of NPM1 gene mutations in childhood acute myeloid leukemia, with emphasis on cytogenetically normal AML. Leukemia. 2009;23:262-270.

7. Falini B, Nicoletti I, Martelli MF, Mecucci C. Acute myeloid leukemia carrying cytoplasmic/mutated nucleophosmin (NPMc+ AML): Biologic and clinical features. Blood. 2007;109:874-885.

8. Miyagawa K, Hayashi Y, Fukuda T, Mitani K, Hira $\mathrm{H}$, Kamiya K. Mutations of the WT1 gene in childhood nonlymphoid hematological malignancies. Genes Chromosomes Cancer. 1999;25:176-183.

9. King-Underwood L, Pritchard-Jones K. Wilms' tumor (WT1) gene mutations occur mainly in acute myeloid leukemia and may confer drug resistance. Blood. 1998;91:2961-2968.

10. Keilholz U, Menssen HD, Gaiger A, et al. Wilms' tumour gene 1 (WT1) in human neoplasia. Leukemia. 2005;19:1318-1323.

11. Ellisen LW, Carlesso N, Cheng T, Scadden DT, Haber DA. The Wilms tumor suppressor WT1 directs stage-specific quiescence and differentiation of human hematopoietic progenitor cells. EMBO J. 2001;20:1897-1909.

12. Summers K, Stevens J, Kakkas I, et al. Wilms' tumour 1 mutations are associated with FLT3-ITD and failure of standard induction chemotherapy in patients with normal karyotype AML. Leukemia. 2007;21:550-551; author reply 552

13. Paschka P, Marcucci G, Ruppert AS, et al. Wilms tumor 1 gene mutations independently predict poor outcome in adults with cytogenetically normal acute myeloid leukemia: A Cancer and Leukemia Group B Study. J Clin Oncol. 2008;26: 4595-4602.

14. Virappane P, Gale R, Hills R, et al. Mutation of the Wilms' tumor 1 gene is a poor prognostic factor associated with chemotherapy resistance in normal karyotype acute myeloid leukemia: The United Kingdom Medical Research Council Adult Leukaemia Working Party. J Clin Oncol. 2008;26: 5429-5435.

15. Kaspers GJ, Veerman AJ, Pieters R, et al. Mononuclear cells contaminating acute lymphoblastic leukaemic samples tested for cellular drug resistance using the methyl-thiazol-tetrazolium assay. Br J Cancer. 1994;70:1047-1052.

16. Van Vlierberghe $P$, van Grotel M, Beverloo HB, et al. The cryptic chromosomal deletion 
del(11)(p12p13) as a new activation mechanism of LMO2 in pediatric T-cell acute lymphoblastic leukemia. Blood. 2006;108:3520-3529.

17. Kardos G, Zwaan CM, Kaspers GJ, et al. Treatment strategy and results in children treated on three Dutch Childhood Oncology Group acute myeloid leukemia trials. Leukemia. 2005;19: 2063-2071.

18. Creutzig U, Zimmermann M, Ritter J, et al. Treatment strategies and long-term results in paediatric patients treated in four consecutive AML-BFM trials. Leukemia. 2005;19:2030-2042.

19. Gibson BE, Wheatley K, Hann IM, et al. Treatment strategy and long-term results in paediatric patients treated in consecutive UK AML trials. Leukemia. 2005;19:2130-2138.

20. Willasch A, Coliva T, Kalinova M, et al. A common multicenter non-commercial approach for quantitative MRD detection by WT1 gene expression PCR. Bone Marrow Transplant. 2008;41:S296.

21. Kreuzer KA, Saborowski A, Lupberger J, et al. Fluorescent 5 '-exonuclease assay for the absolute quantification of Wilms' tumour gene (WT1) mRNA: Implications for monitoring human leukaemias. Br J Haematol. 2001;114:313-318.
22. Barjesteh van Waalwijk van Doorn-Khosrovani S, Erpelinck C, Meijer J, et al. Biallelic mutations in the CEBPA gene and low CEBPA expression levels as prognostic markers in intermediate-risk AML. Hematol J. 2003;4:31-40.

23. Kiyoi H, Naoe T, Yokota S, et al. Internal tandem duplication of FLT3 associated with leukocytosis in acute promyelocytic leukemia. Leukemia Study Group of the Ministry of Health and Welfare (Kohseisho). Leukemia. 1997;11: 1447-1452.

24. Yamamoto $\mathrm{Y}$, Kiyoi H, Nakano Y, et al. Activating mutation of D835 within the activation loop of FLT3 in human hematologic malignancies. Blood. 2001;97:2434-2439.

25. Balgobind $B V$, Van Vlierberghe $P$, van den Ouweland AM, et al. Leukemia-associated NF1 inactivation in patients with pediatric T-ALL and AML lacking evidence for neurofibromatosis. Blood. 2008;111:4322-4328.

26. Caligiuri MA, Strout MP, Oberkircher AR, Yu F, de la Chapelle A, Bloomfield CD. The partial tandem duplication of ALL1 in acute myeloid leukemia with normal cytogenetics or trisomy 11 is restricted to one chromosome. Proc Natl Acad Sci U S A. 1997; $94: 3899-3902$.
27. Little M, Wells C. A clinical overview of WT1 gene mutations. Hum Mutat. 1997;9:209-225

28. Gaidzik V, Habdanu M, Moschny S, et al. Clinical impact of WT1 mutations in the context of other molecular markers in cytogenetically normal acute myeloid leukemia: a study of the GermanAustrian AML study group (AMLSG) [abstract]. Blood. 2007;110:114a.

29. Meshinchi S, Alonzo TA, Stirewalt DL, et al. Clinical implications of FLT3 mutations in pediatric AML. Blood. 2006;108:3654-3661.

30. Meshinchi S, Stirewalt DL, Alonzo TA, et al. Structural and numerical variation of FLT3/ITD in pediatric AML. Blood. 2008;111:4930-4933.

31. Van Dijk JP, Knops GH, Van De Locht LT, et al. Abnormal WT1 expression in the CD34-negative compartment in myelodysplastic bone marrow. Br J Haematol. 2002;118:1027-1033.

32. European Leukemia Information Center (ELIC). Information about Project 12: MRD. http:// www.leukemia-net.org/content/e62/e892/e893/ index_eng.html. Accessed September 7, 2009.

33. Yang L, Han Y, Suarez Saiz F, Minden MD. A tumor suppressor and oncogene: the WT1 story. Leukemia. 2007;21:868-876. 